\title{
Estatinas: Características y Efectos sobre el Control Lipídico en el Niño y Adolescente Obeso
}

\author{
Emilio González Jiménezª, Judit Álvarez Ferre ${ }^{b}$
}

\begin{abstract}
a Departamento de Enfermería. Facultad de Ciencias de la Salud. Universidad de Granada. Granada (España).

b Unidad de Hospitalización Crónica. Hospital Universitario San Rafael. Granada (España).

Correspondencia: Emilio González Jiménez, Departamento de Enfermería, Facultad de Ciencias de la Salud, Avda. de Madrid, s/n, 18071 - Granada (España). Correo electrónico: emigoji@ correo.ugr.es.
\end{abstract}

Recibido el 20 de septiembre de 2010.

Aceptado para su publicación el 15 de diciembre de 2010.

\section{RESUMEN}

La obesidad en niños y adolescentes representa un problema emergente de salud pública, siendo el trastorno nutricional y metabólico más prevalente en los países desarrollados. Paralelo a este incremento de obesidad, han hecho su aparición dislipemias a edades cada vez más tempranas. En la última década se han producido novedosos avances en el tratamiento de estas alteraciones lipídicas, fundamentalmente debido a la introducción de las estatinas en su terapia. Su utilidad y eficacia ampliamente demostrada en adultos parece ser igualmente aplicable en el tratamiento de las hipercolesterolemias durante la infancia. En la actualidad son seis ya las estatinas utilizadas en población infantil, habiendo sido analizada su actividad en niños y jóvenes a través de ensayos clínicos, en los que se concluye su eficacia y seguridad para reducir las concentraciones tanto de colesterol total como de colesterol en lipoproteínas de baja densidad (c-LDL) en sangre. El objetivo de este trabajo ha sido ofrecer una revisión actualizada sobre las características de éstas y sus efectos en la mejora clínica de los niveles de lípidos en sangre en niños y adolescentes con sobrepeso y obesidad.

Palabras clave. Obesidad, Niño, Estatinas, Hiperlipidemias, Arterioesclerosis.

\section{ABSTRACT}

Statins: Characteristics and Effects on lipid control in obese children and adolescents. Obesity in children and adolescents is an emerging public health problem, with nutritional and metabolic disorder being most prevalent in developed countries. Parallel to this increase in obesity, dyslipidemia is occurring at increasingly early ages. The last decade has seen advances in novel treatments for these lipid abnormalities, mainly due to the introduction of statin therapy. Its utility and effectiveness amply demonstrated in adults appears to be equally applicable in the treatment of hypercholesterolemia in childhood. Currently there are six statins used in children. Their activity in children and adolescents has been studied in clinical trials, which determined their efficacy and safety to reduce both total cholesterol and low density lipoprotein cholesterol (LDL-C) levels. The aim of this study was to provide an update on the characteristics of these statins and their effects on clinical improvement of blood lipid levels in overweight and in obese children and adolescents.

Key words. Obesity, Child, Statins, Hyperlipidemias, Atherosclerosis.

\section{INTRODUCCIÓN}

La terapia farmacológica de las alteraciones lipídicas, especialmente el incremento en sangre de las concentraciones de lipoproteínas de baja densidad (c-LDL), ha presentado importantes avances en su abordaje terapéutico'. En la actualidad, existe evidencia científica suficiente como para afirmar que el proceso arteriosclerótico se inicia ya desde la infancia y que su progresión y agravamiento se ven favorecidos por el desarrollo y mantenimiento de estados de hiperlipidemias ${ }^{2}$.

Por otra parte, en los últimos años y de manera solapada al incremento de la tasas de sobrepeso y obesidad juveniles se ha verificado un aumento casi paralelo de las anomalías lipídicas entre dicha población juvenil, con el considerable riesgo cardiovascular que ello supone en etapas posteriores de la vida $^{3}$.

Como resultado de este riesgo, en la actualidad y a pesar de existir cierto grado de controversia respecto del uso de fármacos para corregir las disli- 
pemias en los niños, son numerosos los estudios que sugieren resultados positivos en términos de efectividad y seguridad en pacientes no adultos. El objetivo de este trabajo ha sido analizar la evidencia científica disponible sobre los posibles efectos beneficiosos de las estatinas a nivel de anomalías lipídicas en niños con problemas de sobrepeso/ obesidad y su relación con el proceso arteriosclerótico.

\section{IMPORTANCIA E INDICACIONES DE USO EN PACIENTES PEDIÁTRICOS}

Por término general, entre la población infantil y juvenil el trastorno lipídico más prevalente es la hiperlipidemia primaria ${ }^{4}$. Se trata pues de anormalidades genéticas que conducen a alteraciones en el metabolismo de los lípidos ${ }^{5,6}$, siendo la más común la hipercolesterolemia familiar monogénica (HFM), de carácter autosómico dominante, que cursa con elevaciones de c-LDL, si bien en ocasiones se observan igualmente concentraciones elevadas de triglicéridos y bajas de colesterol, concretamente de lipoproteínas de alta densidad (c-HDL). El gen implicado en dicho proceso se ubica en el brazo corto del cromosoma ${ }^{7}$. Asímismo, se han identificado más de 850 mutaciones del mismo en individuos con hipercolesterolemia familiar monogénica (HFM). No obstante, además de las hiperlipidemias primarias, existen otras que aparecen en diferentes situaciones clínicas y que también podrían requerir tratamiento, sobre todo por aparecer asociadas a otros factores de riesgo cardiovascular como hipertensión, alteraciones del metabolismo de la glucosa y obesidad. Según datos del estudio Bogalusa Heart Study, ya en niños y adolescentes con estados de obesidad leve - moderada se observan niveles superiores de insulina, c-VLDL y c-LDL y menos $\mathrm{C}-\mathrm{HDL}^{8}$. Estos hallazgos a menudo han sido reportados fundamentalmente entre sujetos adultos. Estas elevaciones de los niveles séricos de lípidos y lipoproteínas en los niños y adolescentes van a ser los responsables en última instancia de las complicaciones cardiovasculares en etapas posteriores de la vida, pero como se ha reseñado tienen su origen en alteraciones lipídicas instauradas ya desde la más temprana infancia.

\section{EL PROCESO ARTERIOESCLERÓTICO Y SU ME- TODOLOGÍA ACTUAL DE ESTUDIO EN NIÑOS}

En la actualidad se acepta por la comunidad científica cómo la enfermedad cardiovascular arterioesclerótica tiene su inicio ya en la infancia, para continuar haciéndose progresiva con los años ${ }^{9,10}$. Este proceso se inicia con el desarrollo temprano de la denominada estría lipídica, constituida esencial- mente por un cúmulo de macrófagos cargados de lípidos y adheridos a la túnica íntima arterial. Con el paso del tiempo y a medida que los sujetos avanzan en edad, el depósito lipídico aumenta, asociado a una proliferación de los macrófagos y células del músculo liso, las cuales iniciarán un proceso de migración hacia las túnicas íntima y media arteriales formando finalmente una placa fibrosa. Con el tiempo, esta placa se desestructura pudiendo llegar a romperse e iniciando con ello el proceso de formación del trombo.

Así, según datos del estudio PDAY, concentraciones elevadas de colesterol junto con incrementos en las cifras de presión arterial guardaban una estrecha relación con el desarrollo y grado de extensión de las estrías grasas y placas fibrosas en sujetos de corta edad ${ }^{11}$. Por su parte, el ya mencionado estudio Bogalusa Heart ${ }^{12}$ puso de manifiesto cómo las estrías grasas se encontraban ya presentes en el $50 \%$ de los individuos en la infancia y hasta en un $85 \%$ de los adolescentes. Además, en aras de profundizar más en la etiopatogenia del proceso de arteriosclerosis infantil y juvenil se estableció una correlación entre el grado de desarrollo y extensión de las lesiones arterioscleróticas con los niveles circulantes de c-LDL, triglicéridos, presión arterial e índice de masa corporal de los sujetos, siendo los resultados encontrados absolutamente concluyentes y significativos, y mostrando una relación estrecha entre las variables citadas.

Otro estudio, The Child and Adolescent Trial for Cardiovascular Health (CATCH), en el que se llevó a cabo una valoración del sobrepeso y obesidad para analizar su relación con factores de riesgo cardiovascular en niños de entre 9 y 11 años, puso de manifiesto cómo aquellos niños con sobrepeso (IMC $\geq$ percentil 85 ) presentaban niveles séricos más elevados de colesterol total, $A$ po $B$, de presión arterial sistólica y en cambio los más bajos de c$\mathrm{HDL}^{13}$.

Estos datos indican el extremado riesgo en salud que la presencia elevada en sangre de lipoproteínas y colesterol supone para sujetos de corta edad. No obstante, los hallazgos de lesiones arterioscleróticas a edades tempranas van mucho más allá, habiendo sido descritos casos de lesiones arterioscleróticas en fetos autopsiados, eso sí, descendientes de madres hipercolesterolémicas. Esta circunstancia parece indicar cómo exposiciones prolongadas durante el período fetal intrautero a condiciones de hiperlipemia materna pueden acabar generando procesos arterioscleróticos en el feto.

Como resultado y ante la evidencia de tales hallazgos podemos plantear cómo este proceso manifies- 
ta tener sus primeros pasos ya en la más temprana infancia ${ }^{14}$. No obstante, debido a la baja mortalidad de pacientes pediátricos por esta causa y a las dificultades que supone el estudio de los factores de riesgo en los sujetos autopsiados, se ha convertido en una necesidad indagar en otras técnicas y procedimientos no invasivos que posibiliten el estudio in vivo de estas lesiones y su evolución en el paciente pediátrico ${ }^{15}$.

Esta circunstancia y su gravedad han motivado el interés de la comunidad científica por avanzar en el análisis profundo y en la génesis de estos procesos. De este modo, en los últimos años ha tenido lugar un importante desarrollo a nivel de métodos de estudio del proceso arteriosclerótico en niños y adolescentes. Los métodos desarrollados a este respecto son fundamentalmente dos, el estudio de las anomalías en la estructura vascular y el estudio de anomalías en la función vascular. El primero consiste en la medición del grosor de las capas íntima y media arterial a nivel de localizaciones concretas en las arterias carótidas. Se sabe cómo el grosor de ambas capas se ve incrementado a medida que aumentan los factores de riesgo cardiovascular ${ }^{16,17}$.

El segundo método cobra especial interés en el estudio de alteraciones a nivel de la función vascular. Dentro de este procedimiento destaca la dilatación mediada por flujo (DMF), técnica a través de la cual es posible conocer y valorar de forma precisa las propiedades y naturaleza funcional de las arterias, valorando con ello su rigidez y capacidad de distendir. En este sentido un aspecto valorable mediante este procedimiento será la capacidad adaptativa o de vasodilatación del vaso frente a determinados estímulos. Para llevar a cabo esta valoración en niños, por término general suele utilizarse la arteria braquial siguiendo el estándar existente hasta el momento ${ }^{18}$. Este procedimiento estándar implica la utilización de un manguito de medición de presión arterial. A continuación y mediante su desinflado, aprovechando el incremento de flujo ocasionado en el territorio de la arteria braquial, se procederá a la valoración del estrés generado en el endotelio de dicho vaso.

El incremento de flujo sanguíneo en la arteria producirá un estrés a nivel del endotelio. Esta situación cursará a su vez con la síntesis de óxido nítrico, molécula que inducirá un importante efecto de vasodilatación. Basándose en esta fisiología, el objetivo de la técnica consiste en medir la luz arterial (en su eje longitudinal) antes e inmediatamente después del incremento del flujo sanguíneo para estimar con ello el incremento del diámetro del vaso producido por el efecto de vasodilatación mediado por el óxido nítrico. En aquellas circunstancias en las que el vaso y su endotelio se encuentren afectados por causas o factores de riesgo, la respuesta adaptativa de vasodilatación se verá disminuida. Ello como resultado de unas arterias más rígidas y con menor capacidad de adaptación.

El empleo de esta técnica posibilita el análisis y seguimiento del proceso de arterioesclerosis en niños. Esta circunstancia reviste gran importancia si consideramos la oportunidad que se nos brinda para proceder a la adopción de medidas preventivas orientadas a disminuir los riesgos de cara a la edad adulta.

\section{RECOMENDACIONES ACTUALES EN LA TERA- PIA DE LAS HIPERCOLESTEROLEMIAS}

En la actualidad, el tratamiento a seguir para la hipercolesterolemia en niños pasa por las recomendaciones del National Cholesterol Education Program (NCEP). Desde él y entre las premisas que se proponen destaca la instauración temprana de una dieta apropiada y asociada a un cambio en los estilos de vida, fundamentalmente este último mediado por un incremento del grado de actividad física ${ }^{19}$.

Desde un punto de vista dietético cabe resaltar en el momento actual la importancia del consumo de esteroles vegetales. Estas sustancias también denominadas fitoesteroles pertenecen a la familia de los triterpenos y son esteroles derivados de plantas con estructuras análogas a las del colesterol propio de los vertebrados, pero que incluyen además un grupo metilo o etilo en el carbono veinticuatro ${ }^{20}$. Se han descrito más de 200 tipos diferentes de esteroles vegetales en diferentes especies de plantas, siendo el más abundante el sitosterol o b-sitosterol, seguido por el campesterol y el estigmasterol ${ }^{21}$. En relación a su biosíntesis, cabe destacar cómo ésta resulta muy diferente en animales y en plantas. En el caso de los humanos, nuestro organismo no puede sintetizar esteroles vegetales, por lo que su ingesta mediante la dieta será la única fuente. En cambio, en las plantas, los esteroles vegetales son sintetizados también a partir de acetato a través de escualeno ${ }^{22}$.

Las fuentes más importantes de esteroles son los aceites vegetales, aceites como los de maíz, girasol, soja y colza, los cuales contienen entre un $0,1 \%$ y $0,8 \%$ de esteroles. Una persona que consuma 30 $\mathrm{g}$ de aceite de maíz al día estaría ingiriendo alrededor de $300 \mathrm{mg}$ de esteroles vegetales, cantidad que se ha demostrado posee eficacia a la hora de reducir la absorción orgánica de colesterol ${ }^{23}$. En el caso de patrones dietéticos occidentales, la ingesta de estas sustancias se estima en unos 150-400 mg diarios, aproximadamente la misma que la ingesta 
de colesterol. Únicamente en algunas dietas vegetarianas y en modo especial en la dieta japonesa su ingesta puede resultar aún mayor pudiendo alcanzar los $300-500 \mathrm{mg} / \mathrm{dí}^{24}$.

Únicamente en aquellos pacientes con edades superiores a los 8-10 años en los que la terapia con esteroles no resultase efectiva y con concentraciones de c-LDL superiores a $190 \mathrm{mg} / \mathrm{dl}$, o bien mayores de $160 \mathrm{mg} / \mathrm{dl}$ pero con antecedentes familiares positivos para enfermedades cardiovasculares tempranas $u$ factores de riesgo como obesidad, diabetes e hipertensión, estaría recomendado el tratamiento farmacológico. Dicha terapia hasta el momento actual contempla el uso de fármacos como la colestiramina o el colestipol a dosis que pueden oscilar entre los 4-5 g/día hasta los $20 \mathrm{~g} /$ día. Ambos fármacos funcionan interrumpiendo el ciclo enterohepático e impidiendo la reabsorción de colesterol y ácidos biliares. No obstante, los resultados obtenidos por el momento en pacientes pediátricos y adolescentes son poco alentadores, resultado fundamentalmente de sus importantes efectos secundarios a nivel digestivo dada su intensa sintomatología de estreñimiento, meteorismo y dolor cólico. No obstante, a pesar de mantener un adecuado cumplimiento en su administración por parte del paciente, las mejoras en términos de reducción de la concentración de c-LDL resultan parciales, alcanzando reducciones únicamente del $15-20 \%{ }^{25}$

Teniendo en cuenta lo anterior, parece racional el interés inusitado entre los pediatras por las estatinas. No obstante, previo a la incorporación de todo nuevo fármaco a la práctica clínica resulta esencial demostrar la seguridad de uso, más aún cuando la población a la que se dirige es la pediátrica.

\section{MECANISMO DE ACCIÓN DE LAS ESTATINAS}

El efecto hipocolesterolémico de los esteroles vegetales ha sido ampliamente documentado y revisa$\mathrm{do}^{26}$. Las primeras observaciones del efecto hipocolesterolémico de los fitoesteroles datan de 50 años atrás, a partir de la observación de cómo la alimentación de pollos con semillas de soja producía un descenso en las concentraciones de colesterol sanguíneo. Estos efectos fueron igualmente observados en humanos en estudios posteriores ${ }^{27}$.

Las estatinas disminuyen la síntesis endógena de colesterol mediante inhibición competitiva de la en-

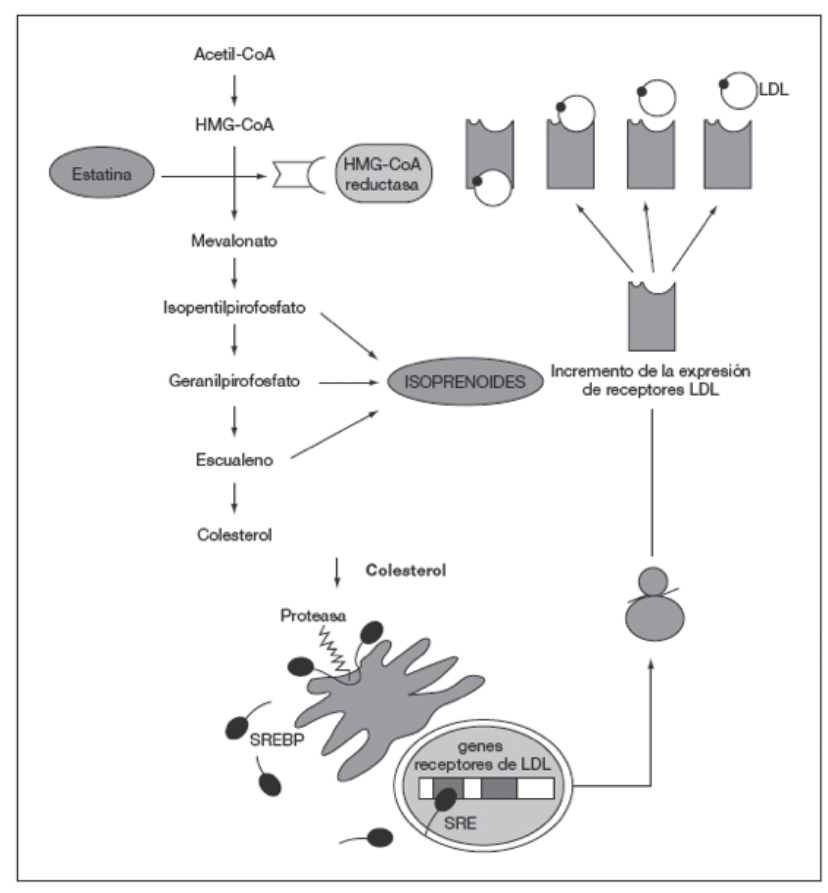

Figura 1. Mecanismo de acción de las estatinas. Adaptado de: Tapia et al ${ }^{42}$. HMG-CoA: hidroximetilglutaril-coenzima A. LDL: lipoproteínas de baja densidad. SRE: elemento regulador de esteroles. SREBP: proteínas de unión al elemento regulador de esteroles. 
zima HMG-CoA reductasa, enzima limitante en la biosíntesis del colesterol. Ocupan una porción del receptor bloqueando la unión del sustrato al lugar de actividad enzimática ${ }^{28}$ Como ilustra la figura 1 , la deplección de colesterol activa una proteasa, la cual escinde proteínas de unión al elemento regulador de esteroles (SREBP) desde el retículo endoplásmico. Los SREBP son traslocados al núcleo, aumentando la expresión de genes de receptores de LDL, lo que origina un incremento en la endocitosis mediada por receptor de LDL con la subsiguiente disminución sérica de la concentración de LDL. La inhibición de HMG-CoA reductasa también reduce las concentraciones intracelulares de isoprenoides, intermediarios en la biosíntesis de colesterol ${ }^{28}$. Un beneficio adicional de la terapia con estatinas consiste en modificar los porcentajes de las subfracciones de LDL, y da como resultado un perfil menos aterogénico al disminuir el porcentaje de partículas de LDL pequeñas y densas ${ }^{29}$. Además, alguna de ellas como la atorvastatina, también reduce la producción de las lipoproteínas de muy baja densidad (VLDL) a través de un efecto mediado por la secreción hepática de apo-B, lo cual se asocia con una disminución de la actividad de la HMG-CoA reductasa después del tratamiento farmacológico.

Diversos estudios han mostrado que 2 a $3 \mathrm{~g}$ de estanoles vegetales, formando parte de una dieta moderada en grasas, reducen perceptiblemente las concentraciones séricas totales de colesterol total y colesterol LDL sin afectar las concentraciones del colesterol HDL o los triglicéridos ${ }^{30}$.

Respecto de su eficacia en niños en la reducción de las concentraciones de c-LDL, ésta oscila entre el 17 y el $45 \%$ dependiendo del tipo de estatina y dosis utilizada ${ }^{30,31,32}$. Por otra parte, se han verificado otros efectos positivos sobre el c-HDL, originando elevaciones discretas (entre el 1-11\%) del mismo, o reduciendo las concentraciones de triglicéridos hasta en un $17 \%{ }^{33,34}$. Ello, en parte, mediado por una disminución de la síntesis de VLDL. Con todo ello, más relevante aún es el dato de que el tratamiento con estatinas no sólo disminuye las concentraciones de colesterol, sino que además revierte considerablemente los fenómenos de arterioesclerosis, mejorando la dilatación mediada por flujo (DMF), así como reduciendo la progresión del engrosamiento a nivel de la túnica íntima-media en vasos como la carótida $^{35}$.

\section{EFECTOS ADVERSOS DERIVADOS DE SU UTI- LIZACIÓN EN NIÑOS}

Entre los efectos adversos descritos en adultos destacan fundamentalmente eventos de tipo gas- trointestinal tales como estreñimiento, diarrea, meteorismo, dolor abdominal y dispepsia. Igualmente han sido descritos dolores musculares (mialgias) y alteraciones hepáticas que van a cursar con elevación de las enzimas hepáticas. Otros síntomas comunes son cefaleas, astenia, exantemas cutáneos, rabdomiolisis, prurito y trastornos del sueño. Sin embargo, de todos ellos, el más grave es la rabdomiolisis, circunstancia que puede verse agravada si estos fármacos se administran de manera conjunta con otros metabolizados vía citocromo P-450 como la ciclosporina, eritromicina o el ketoconazol. En el caso de población infantil y juvenil, y a diferencia de los adultos, no han sido descritos casos de miositis, miopatía o rabdomiólisis. Se han encontrado algunos casos de elevaciones asintomáticas de la creatinfosfocinasa y ciertas enzimas hepáticas, pero que han sido corregidos mediante ajustes en las dosis y en todos los casos sin necesidad de suspender el tratamiento. Tampoco se han encontrado trastornos del crecimiento o el desarrollo puberal.

\section{UTILIZACIÓN EN NIÑOS Y ADOLESCENTES}

La utilización de estatinas en el tratamiento de las hiperlipemias en el paciente pediátrico representa aún en la actualidad un modelo terapéutico controvertido.

Resultados obtenidos en ensayos clínicos con estatinas en niños y adolescentes con historia familiar de hipercolesterolemia grave indican que los efectos adversos no parecen ser mayores de los apreciados en adultos. En la actualidad, son seis las estatinas utilizadas en población infantil y juvenil (lovastatina, fluvastatina, pravastatina, simvastatina, atorvastatinan y rosuvastatina), si bien es cierto que sólo cuatro de ellas (lovastatina, simvastatina, pravastatina y atorvastatina) han sido aceptadas por la US Food and Drug Administration (FDA) para uso en pacientes pediátricos ${ }^{36,37}$.

Importantes revisiones sobre su utilización en la infancia concluyen que, a la vez que seguras, son muy eficaces para reducir las concentraciones tanto del colesterol total como del c-LDL ${ }^{38,39}$. Dicha reducción, aunque sujeta a oscilaciones (17-45\% respecto de las concentraciones iniciales), ha dependido del tipo de estatina utilizada y dosis empleada. Con todo ello, las mejoras más importantes observadas en términos de reducción de colesterol y c-LDL han venido de la mano de la atorvastatina a dosis de 10-40 mg/día ${ }^{40}$.

Finalmente, teniendo en cuenta todo lo expuesto y a modo de reflexión, cabría plantearse si los esteroles vegetales como productos destinados a pa- 
cientes con hipercolesterolemia sobrepasan, o no, el concepto generalmente aceptado de alimento funcional (alimentos con efectos beneficiosos para la salud que superan el ámbito nutricional y contribuyen a la prevención o protección frente a ciertas enfermedades).

No obstante, teniendo en cuenta sus efectos beneficiosos tanto en adultos como en niños cabe plantear cómo éstos van más allá del mero ámbito de la protección frente a enfermedades cardiovasculares en sujetos con problemas de sobrepeso-obesidad. En esta situación, cabría enmarcarlos como otra alternativa a la terapéutica actual del paciente pediátrico y adolescente con dislipemias.

\section{BIBLIOGRAFÍA}

1. Ferranti S, Ludwig DS. Storm over statins. The controversy surrounding pharmacologic treatment of children. $\mathrm{N}$ Eng $\mathrm{J}$ Med. 2009; 359:1309-12.

2. Spiotta RT, Luma GB. Evaluating obesity and cardiovascular risk factors in children and adolescents. Am Fam Physician. 2008; 78(9):1052-58.

3. Kwiterovich PO. Recognition and management of dyslipidemia in children and adolescents. J Clin Endocrinol Metab. 2008; 93:4200-09.

4. McCrindle BW. Hyperlipidemia in children. Thromb Res. 2006; 118:49-58.

5. Civeira F. Guidelines for the diagnosis and management of heterozygous familial hypercholesterolemia. Atherosclerosis. 2004; 173:55-68.

6. Pullinger CR, Eng C, Salen G, Shefer S, Batta AK, Erickson SK, et al. Human cholesterol 7 alpha hydroxylase (CYP7A1) deficiency has a hypercholesterolemic phenotype. J Clin Invest. 2002; 110:109-17.

7. Istvan ES, Deisenhofer J. Structural mechanism for statin inhibition of HMG-CoA reductasa. Science. 2001; 292:1160.

8. Lu K, Lee MH, Hazard S, Brooks-Wilson A, Hidake H, Kojima $\mathrm{H}$, et al. Two genes that map to STSL locus cause sitosterolemia: Genomic structure and spectrum of mutations involving sterolin-1 and sterolin-2, encoded by ABCG5 and ABCG8, respectively. Am J Hum Genet. 2001; 69:278-90.

9. De Jongh S, Lilien MR, Bakker HD, Hutten BA, Kastelein JJP, Stroes ESG. Family history of cardiovascular events and endothelial dysfunction in children with hypercholesterolemia. Atherosclerosis. 2002; 163:193-97.

10. Wiegman A, De Groot E, Hutten BA, Rodenburg J, Gort J, Bakker HD, et al. Arterial intima-media thickness in children heterozygous for familial hypercholesterolemia. Lancet. 2004; 363:369-70.

11. Relationship of atherosclerosis in young men to serum lipoprotein colesterol concentrations and smoking: A preliminary report from the Pathobiological Determinants of Atherosclerosis in Youth (PDAY) Research Group. JAMA. 1990; 264:3018-24.

12. Newman WP, Freedman DS, Voors AW, Gard PD, Srinivasan SR, Cresanta JL, et al. Relation of serum lipoprotein levels and systolic blood pressure to early atherosclerotisis: The Bogalusa Heart Study. N Engl J Med. 1986; 314:13844.

13. Tojo Sierra R, Leis Trabazo R. La obesidad, un problema emergente en pediatría Conferencia inaugural del VIII Congreso Nacional de la Sociedad Española de Nutrición, Mur- cia, 24-27 de octubre de 2001. Nutr Hosp. 2002; 17: 75-9.

14. Skilton MR, Celermajer DS. Endothelial dysfunction and arterial abnormalities in childhood obesity. Int J Obes. 2006; 30:1041-49.

15. Li S, Chen W, Srinivasav SR, Bond MG, Tang R, Urbina EM, et al. Childhood cardiovascular risk factors and carotid vascular changes in adulthood: The Bogalusa Heart Study. JAMA. 2003; 290:2271-76.

16. Quyyumi AA. Endothelial functions on health and disease: new insigths into the genesis of cardiovascular disease. Am J Med. 1998; 105:325-95.

17. Corretti MC, Anderson TJ, Benjamin EJ, Celermajer D, Charbonneau F, Creager MA, et al. Guidelines for the ultrasound assessment of endothelial flow-mediated vasodilation of the brachial artery: A report of the International Brachial Artery Reactivity Task Force. J Am Coll Cardiol. 2002; 39:257-65.

18. American Academy of Pediatrics. National Cholesterol Education Program: Report of the Expert Panel on Blood Cholesterol Levels in Children and Adolescents. Pediatrics. 1992; 89:525-84.

19. Ostlund RE. Phytosterols in human nutrition. Annu Rev Nutr. 2002; 22:533-49.

20. Kochhar SP. Influence of processing on sterols of edible vegetable oils. Prog Lipid Res. 1983; 22:161-88.

21. Miettinen TA, Tilvis RS, Kesaniemi YA. Serum plant sterols and colesterol precursors reflect cholesterol absorption and synthesis in volunteers of a randomly selected male population. Am J Epidemiol. 1990; 131:20-31.

22. Ostlund RE, Racette SB, Okeke A, Stenson WF. Phytosterols that are naturally present in commercial corn oil significantly reduce cholesterol absorption in humans. Am J Clin Nutr. 2002; 75:1000-4.

23. Nair PP, Turjman N, Kessie G, Calkins B, Goodman GT, Davidovitz $\mathrm{H}$ et al. Diet, nutrition intake, and metabolismo in populations at high and low risk for colon cancer. Dietary cholesterol, beta-sitosterol, and stigmasterol. Am J Clin Nutr. 1984; 40 (Suppl. 4):927-30.

24. Tonstad S, Knudtzon J, Sivertsen M, Refsum H, Ose L. Efficacy and safety of cholestyramine therapy in peripuberal and prepuberal children with familial hypercholesterolemia. J Pediatr. 1996; 129:42-9.

25. Sanders, DJ, Minter, HJ, Howes, D, Hepburn, PA. The safety evaluation of phytosterol esters. Part 6 . The comparative absorption and tissue distribution of phytosterols in the rat. Food Chem Toxicol. 2000; 38:485-91.

26. Pollak OJ. Reduction of blood cholesterol in man. Circulation. 1953; 7:702-6.

27. Vaughan CJ, Gotto AM, Basson CT. The evolving role of statins in the management of atherosclerosis. J Am Coll Cardiol. 2000; 35:1-10.

28. Otvos JD, Shalaurova I, Freedman DS, Rosenson RS. Effects of pravastatin treatment on lipoprotein subclass profiles and particle size in the PLAC-I trial. Atherosclerosis. 2002; 160:41.

29. Jenkins, DJ, Kendall, CW, Faulkner, D. y cols. A dietary portfolio approach to cholesterol reduction: Combined effects of plant sterols, vegetable proteins, and viscous fibers in hypercholesterolemia. Metabolism. 2002; 51:1596-1604.

30. Rodenburg J, Visser MN, Trip MD, Wiegman A, Bakker HD, Kastelein JJP. The spectrum of statin therapy in hyperlipidemic children. Seminars in Vascular Medicine. 2004; $4: 313-20$

31. Wiegman A, Hutten BA, De Groot E, Rodenburg J, Bakker $\mathrm{HD}$, Buller HR, et al. Efficacy and safety of statin therapy in children with familial hypercholesterolemia: A randomized controlled trial. JAMA. 2004; 292:331-7.

32. McCrindle BW, Ose L, Marais AD. Efficacy and safety of 
atorvastatinin children and adolescents with familial hipercolesterolemia or severe hyperlipidemia: A multicenter, randomized, placebo-controlled trial. J Pediatr. 2003; 142: 74-80.

33. Amundsen AL, Ose L, Nenseter MS, Ntanios FY. Plant sterol ester-enriched spread lowers plasma total and LDL cholesterol in children with familial hypercholesterolemia. Am J Clin Nutr. 2002; 76:338-44.

34. Ketomaki A, Gylling H, Miettinen TA. Effects of plant stanol and sterol esters on serum phytosterols in a family with familial hypercholesterolemia including a homozygous subject. J Lab Clin Med. 2004; 143:255-62.

35. Berenson GS, Srinvasan SR, Bao W. For the Bogalusa Herat Study Association between múltiple cardiovascular risk factors and atherosclerosis in children and young adults. N Engl J Med. 1998; 338:1650-56.

36. De Jongh S, Ose L, Szamosi T, Gagne C, Lambert M, Scott $R$, et al. Efficacy and safety of statin therapy in children with familial hypercholesterolemia: A randomized, double-blind, placebo- controlled trial with simvastatin. Circulation. 2002; 106:2231-37.
37. Shepherd J, Hunninghake DB, Stein EA, Kastelein JJ, Harris, Pears J, et al. Safety of rosuvastatin. Am J Cardiol. 2004; 94:882-88

38. Celermajer DS, Sorensen KE, Gooch VM. Non invasive detection of endothelial dysfunction in children and adults at risck of atherosclerosis. Lancet. 1992; 340:1111-15.

39. Woo KS, Chook P, Yu CW, et al. Overweight in children is associated with arterial endothelial dysfunction and intimamedia thickening. Int J Obes. 2004; 28:852-57.

40. Meyer AA, Kundt G, Steiner M, Schuff-Werner P, Kienast W. Impaired flow-mediated vasodilation, carotid artery intimamedia thickening, and elevated endothelial plasma markers in obese children: the impact of cardiovascular risk factors. Pediatrics. 2006; 117:1560-67.

41. Daniels SR, Greer FR; Committee on Nutrition. Lipid screening and cardiovascular health in childhood. Pediatrics. 2008; 122:198-208.

42. Tapia L, Picazo B, Ruiz C. Uso de estatinas durante la infancia. An Pediatr (Barc). 2008; 68(4):385-92. 